\title{
Afternoon mid-latitude current system and low-latitude geomagnetic field asymmetry during geomagnetic storms
}

\author{
A. Grafe ${ }^{2}$, P. A. Bespalov ${ }^{1}$, V. Y. Trakhtengerts ${ }^{1}$, A. G. Demekhov ${ }^{1}$ \\ ${ }^{1}$ Institute of Applied Physics, 46 Ulyanov St., 603600 Nizhny Novgorod, Russia \\ ${ }^{2}$ GeoForschungsZentrum, Telegrafenberg, D-14473 Potsdam, Germany
}

Received: 30 October 1996 / Revised: 16 April 1997 / Accepted: 12 May 1997

\begin{abstract}
For four geomagnetic storms of middle intensity the relationship between the low-latitude magnetic field asymmetry using ASY indices and the intensity of the auroral eastward and westward electrojet was considered. It was asked whether there exists a connection between ASY and the eastward electrojet. To answer this question equivalent current systems were estimated in mid-latitudes. It was found that the observations obviously show no correlative relationship between the low-latitude magnetic-field asymmetry and the eastward electrojet, whereas one exists between ASY and the westward electrojet. To explain the generally accepted common three-dimensional current system between the partial ring current and the eastward electrojet, a condensor model of the three-dimensional current system was developed. It could be shown that the short periodic variations of the partial ring current are shielded by the condensor and cannot influence the eastward-electrojet current.
\end{abstract}

\section{Introduction}

Since the work of Kamide and Fukushima (1972) it has been generally accepted that in the evening sector the asymmetric ring current is part of a three-dimensional current system containing the eastward auroral electrojet. However, there are some doubts that this conception is correct. Grafe (1977) and Clauer et al. (1981) have shown that the relationship between the intensity of the low-latitude magnetic field asymmetry and the intensity of the westward auroral electrojet is closer than between the asymmetry and the eastward auroral electrojet. This implies that the relationship between DR and DP field variation is far from clear, maybe because the nature of

Correspondence to: A. Grafe (grafe@gfz-potsdam.de) the eastward electrojet itself is not quite clear. It is well known that the westward electrojet can be classified into two different phenomena, the convection westward electrojet and the explosive westward electrojet, caused by two different magnetospheric processes, the largescale direct driven process and the unloading process (Sergeev, 1977; Pytte et al., 1978; Kamide and Vickrey, 1983). In the case of the eastward electrojet, however, it is generally supposed that it is only caused by the direct driven process. Therefore the eastward electrojet will be only a convection electrojet (Baumjohann, 1986). However, Grafe $(1990,1994)$ has shown that the eastward electrojet has also a double nature. During precipitation events in the evening sector the eastward electrojet shows an explosive feature characterized by pi2 pulsations at the beginning of the event. It is surmised that the double nature of the eastward electrojet is the cause of the bad correlation between the ASY- index and AU. For this reason we deal in this paper with an analysis of the relationship between the storm-time asymmetry and substorm equivalent current system. We will show this using examples in the afternoon/evening sector in midand auroral latitudes for various storms. However, for all these investigations we have to consider that the lowlatitude magnetic-field asymmetry cannot be explained alone by an asymmetric ring current; this has been shown by Harel et al. (1981) and Crooker and Siscoe (1981). Referring to them, the region-1 Birkeland current plays an important role in the low-latitude asymmetry. On the other hand Grafe et al. (1996) show that during the recovery phase, especially for median disturbed storms, the low-latitude magnetic field proceeds differently in different local times. The recovery phase typical for the ring current runs differently in different local times. This means that the observed asymmetry of the low-latitude magnetic field during the recovery phase is mainly caused by the local-time differences of the ring-current decay. It will be the task of this work to investigate the relationship between the low-latitude field asymmetry and the mid-latitude current system. 


\section{Observations}

In order to investigate the relationship between the asymmetric disturbance field in low latitudes and the current system in mid-latitudes we have used the magnetic field observations of six observatories in low latitudes shown in Table 1, of the seven EISCAT magnetometer cross stations (Lühr et al., 1984) shown in Table 2, and of 13 observatories in mid-latitudes shown in Table 3. For this investigation we have selected four storms of the last sunspot maximum. The dates are the following: 9-11 April 1990; 9-11 October 1990; 27- 28 November 1990; 23-25 March 1991. For all magnetic observations we have calculated for these storms instant storm-time values. Sq was eliminated. The horizontal magnetic field components were converted into the geomagnetic components. The low-latitude magnetic

Table 1. Low-latitude observatories

\begin{tabular}{llllll}
\hline station & \multicolumn{2}{l}{ geographic coordinates } & & \multicolumn{2}{l}{ geomagnetic coordinates } \\
\cline { 2 - 3 } \cline { 5 - 6 } & $\varphi$ & $\lambda$ & & $\Phi$ & $\Lambda$ \\
\hline Tbilisi & 42.08 & 44.70 & & 36.61 & 122.48 \\
Taschkent & 41.33 & 69.62 & & 32.30 & 144.43 \\
Teoloyucan & 19.75 & 260.82 & & 29.59 & 327.56 \\
Honolulu & 21.32 & 202.00 & & 21.17 & 266.99 \\
Kakioka & 36.23 & 140.18 & & 26.09 & 206.38 \\
San Juan & 18.23 & 293.93 & & 29.90 & 3.20 \\
\hline
\end{tabular}

Table 2. EISCAT magnetometer cross stations

\begin{tabular}{llllll}
\hline station & \multicolumn{2}{l}{ geographic coordinates } & & \multicolumn{2}{l}{ geomagnetic coordinates } \\
\cline { 2 - 3 } \cline { 5 - 6 } & $\varphi$ & $\lambda$ & & $\Phi$ & $\Lambda$ \\
\hline Söröya & 70.54 & 22.22 & & 67.22 & 120.80 \\
Alta & 69.90 & 22.96 & & 66.49 & 120.68 \\
Kevo & 69.76 & 27.01 & & 65.64 & 123.49 \\
Kilpisjärvi & 69.02 & 20.79 & & 66.06 & 118.24 \\
Kautokeino & 69.00 & 23.00 & & 65.78 & 119.54 \\
Muonio & 68.02 & 23.53 & & 64.69 & 119.42 \\
Pello & 66.90 & 24.08 & 63.64 & 118.60 \\
\hline
\end{tabular}

field data were used to estimate the Dst variation and the ASY index as according to Kawasaki and Akasofu (1971), which is the measure of the asymmetry of the magnetic field in low latitudes concerning the local time. ASY describes the difference between the highest and lowest geomagnetic field disturbance values. ASY is measured as a positive value. The Dst- and ASY-indices are represented in Figs. 1-4. The arrows in these figures indicate the selected time moments for which horizontal magnetic field vectors were estimated by use of the midand high-latitude magnetograms. These vectors are storm-time vectors and describe the storm activity after the sudden commencement. Figures 9-12 show the plots of these vectors and additionally the equivalent current systems. The results are more or less quantitative. The time moments were chosen to investigate current systems in mid-latitudes in relation to the low- latitude field asymmetry in the afternoon/evening sector. In Figs.

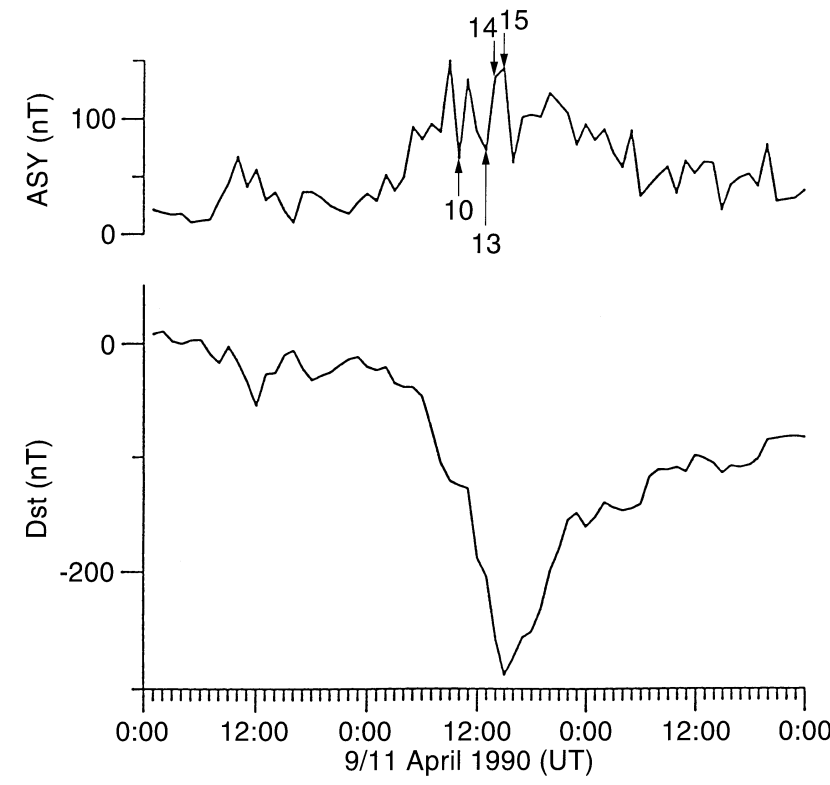

Fig. 1. Dst and ASY values of the geomagnetic $X$-component on 9-11 April 1990

Table 3. Mid-latitude stations

\begin{tabular}{|c|c|c|c|c|}
\hline \multirow[t]{2}{*}{ station } & \multicolumn{2}{|c|}{ geographic coordinates } & \multicolumn{2}{|c|}{ geomagnetic coordinates } \\
\hline & $\varphi$ & $\lambda$ & $\Phi$ & $\Lambda$ \\
\hline Dombas & 62.07 & 9.12 & 62.17 & 100.48 \\
\hline Nurmijärvi & 60.52 & 24.65 & 56.62 & 103.05 \\
\hline Leningrad & 59.95 & 30.70 & 56.17 & 117.70 \\
\hline Borok & 58.03 & 38.97 & 52.95 & 123.50 \\
\hline Brorfelde & 55.62 & 11.67 & 55.54 & 98.71 \\
\hline Moscow & 55.48 & 37.32 & 50.80 & 120.87 \\
\hline Wingst & 53.75 & 9.07 & 54.46 & 94.47 \\
\hline Niemegk & 52.07 & 12.68 & 51.99 & 97.67 \\
\hline Belsk & 51.84 & 20.80 & 50.34 & 104.45 \\
\hline $\mathrm{Kie}$ & 50.72 & 30.30 & 47.50 & 118.56 \\
\hline Lvov & 49.90 & 23.75 & 47.94 & 106.26 \\
\hline Budkov & 49.07 & 14.01 & 49.04 & 96.79 \\
\hline Fürstenfeldbruck & 48.17 & 11.28 & 48.73 & 93.75 \\
\hline
\end{tabular}



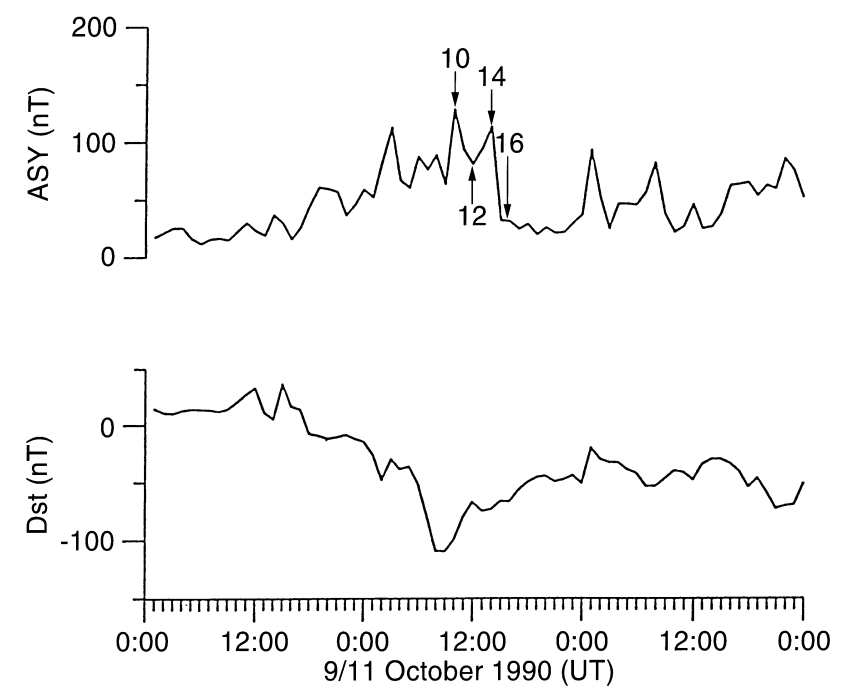

Fig. 2. Dst and ASY values of the geomagnetic $X$-component on 9-11 October 1990
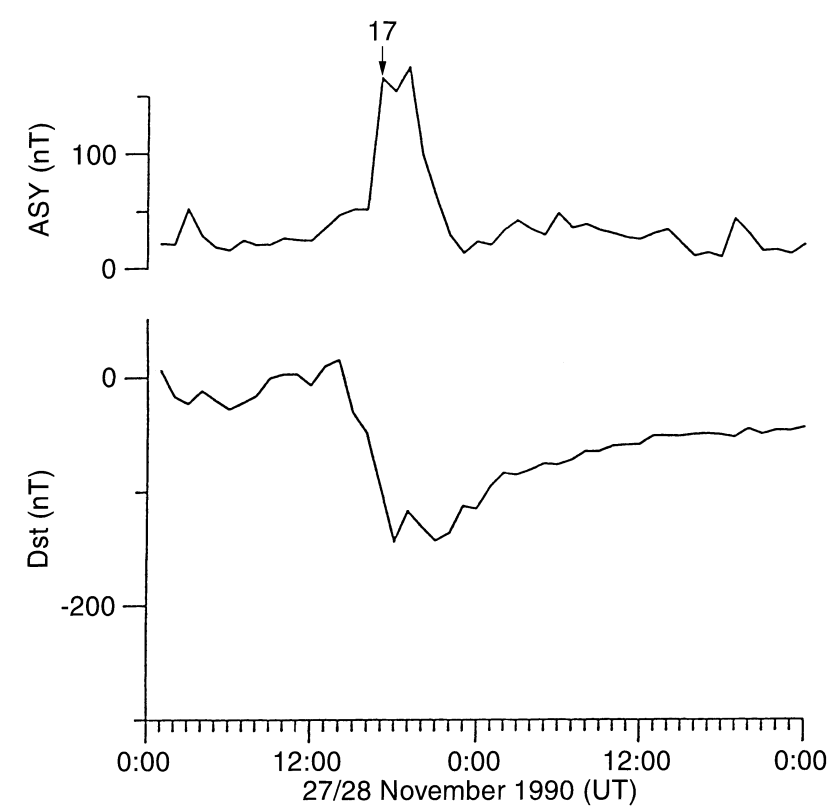

Fig. 3. Dst and ASY values of the geomagnetic $X$-component on $27-$ 28 November 1990

5-8 the X-components of the EISCAT magnetometer cross stations are shown; they show clearly the activity of the eastward- and the westward-directed electrojet of the four storms.

\section{Results of observations}

The essential question to be answered by this investigation is the following: Is there a relationship between the low-latitude magnetic field asymmetry and the smallscale equivalent current system in mid-latitudes? The magnetic-field observations of the four storms shown in Figs. 1-12 will provide us with the answer.

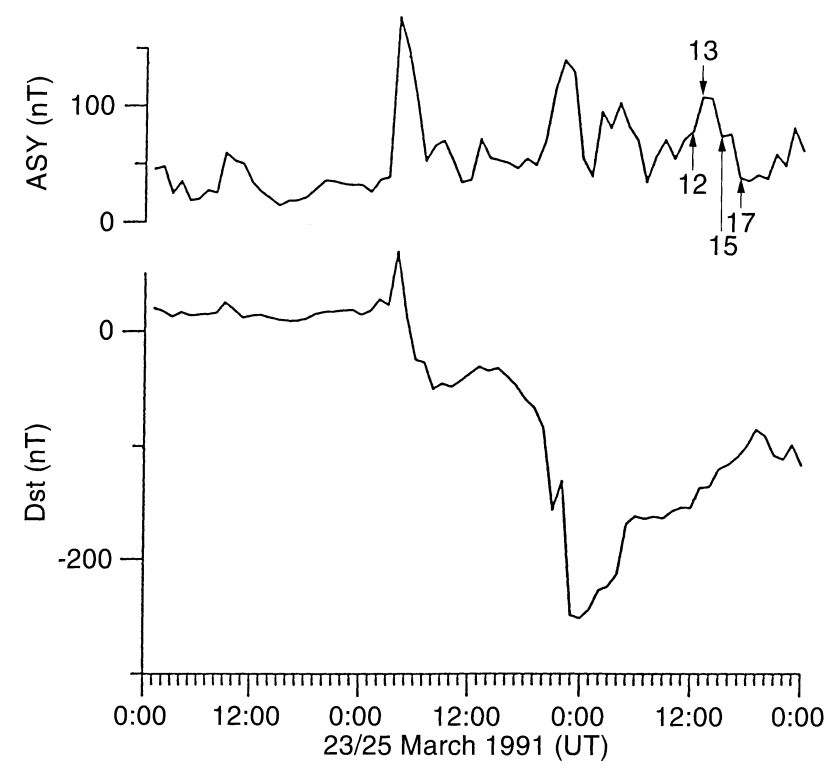

Fig. 4. Dst and ASY values of the geomagnetic $X$-component on 2325 March 1991

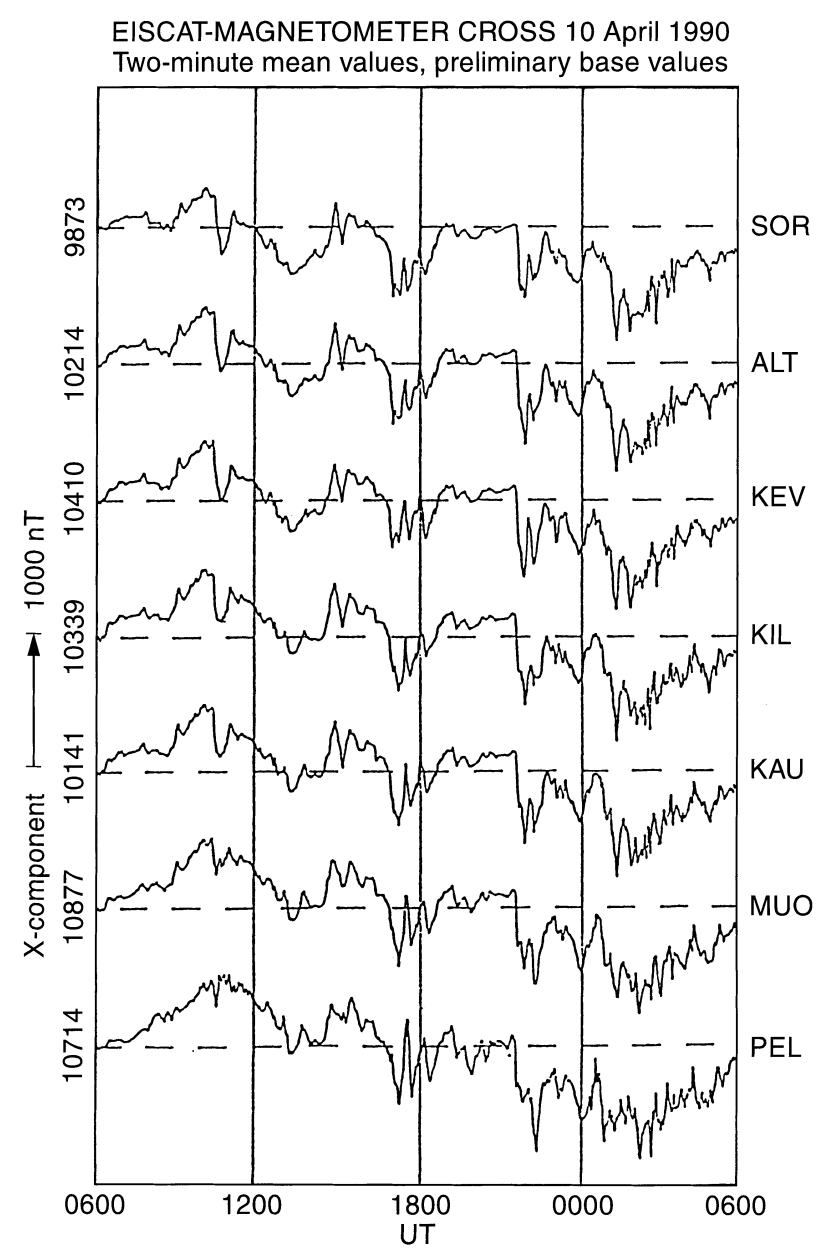

Fig. 5. EISCAT magnetometer data (geographic $X$-component) on 911 April 1990 


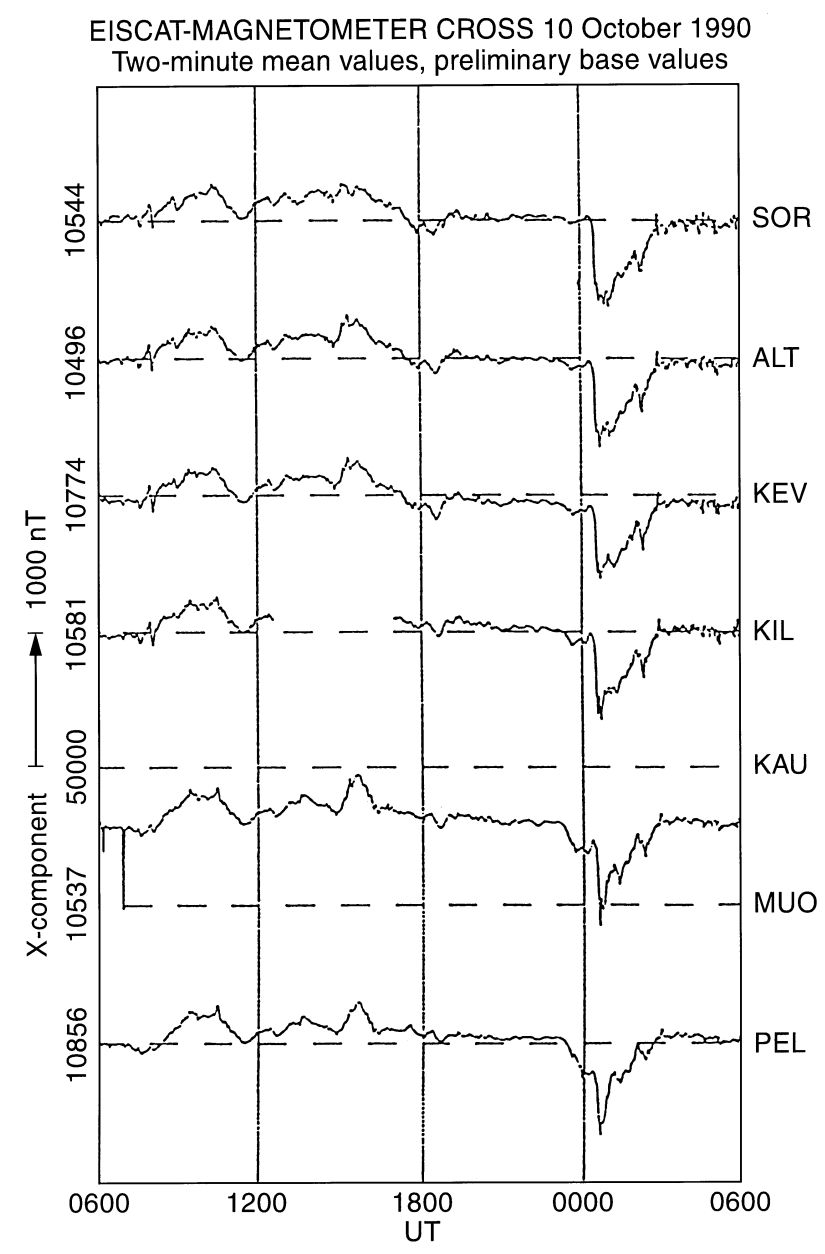

Fig. 6. EISCAT magnetometer data (geographic $X$-component) on 911 October 1990

\subsection{Storm on 10 April 1990}

On this day the maximum of the eastward-electrojet intensity (positive bay, Fig. 5) appeared at 1000 UT. A pronounced current system (Fig. 9) has developed. However, Fig. 1 shows at this time only a low asymmetry of about $60 \mathrm{nT}$. In comparison to other time moments the asymmetry is $>100 \mathrm{nT}$. At $1300 \mathrm{UT}$ the conditions are clearer. The eastward electrojet has disappeared and the asymmetry is low, similar to 1000 UT. But $1 \mathrm{~h}$ later at $1400 \mathrm{UT}$, when the eastward electrojet is also missing and no current system in midlatitudes exists, we observe a high asymmetry of about $140 \mathrm{nT}$. And at $1500 \mathrm{UT}$ for a short time interruption of a new enhanced eastward electrojet by a polewardappearing westward electrojet took place. Simultaneously we observe high asymmetry and a well-pronounced current system. Looking to 0900 and 1100 UT we see at these moments also an overlapping of the eastward electrojet by a poleward westward electrojet. In Fig. 13, as well as the ASY index, the AL and AU (provisional values) are also represented. The correlation between ASY and AL is excellent. From this event we cannot conclude that a relationship between the intensity of the low-latitude magnetic field asymmetry and the mid-

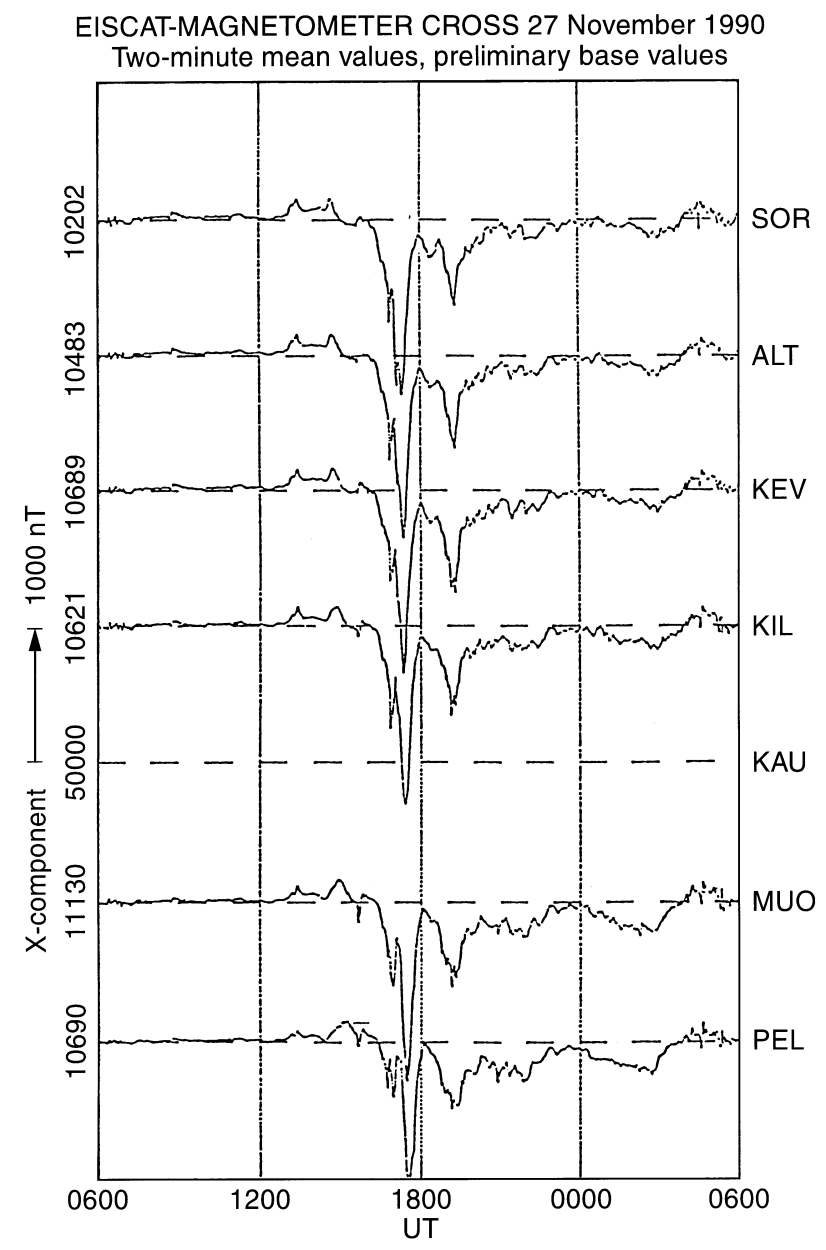

Fig. 7. EISCAT magnetometer data (geographic $X$-component) on 27-28 November 1990

latitude current system in the afternoon/evening sector exists. In contrast, we suppose that there is more of a relationship between the asymmetry and the westwardelectrojet current system.

\subsection{Storm on 10 October 1990}

This storm is the least intensive of the four. Dst maximum is only about $100 \mathrm{nT}$ (Fig. 2). Figure 6 shows that the eastward-electrojet intensity is not high. During the time-interval from 1000 to 1600 UT there was no variation in the well-pronounced current system at midlatitudes (Fig. 10). However, during this time-interval the asymmetry shown in Fig. 2 on the right-hand side changes strongly. The decrease in asymmetry from 1000 to $1600 \mathrm{UT}$ is about $100 \mathrm{nT}$, but the current systems at these two time moments are fairly equal. Therefore, we can also conclude from this interval that obviously no relationship between asymmetry and the eastwardelectrojet current system development in mid-latitudes exists. The positive $X$ component in the afternoon of this event has a character of a convection eastward electrojet. No short periodic sudden positive variations characteristic of an explosive eastward electrojet are recognizable. 
EISCAT-MAGNETOMETER CROSS 25 March 1991 Two-minute mean values, preliminary base values

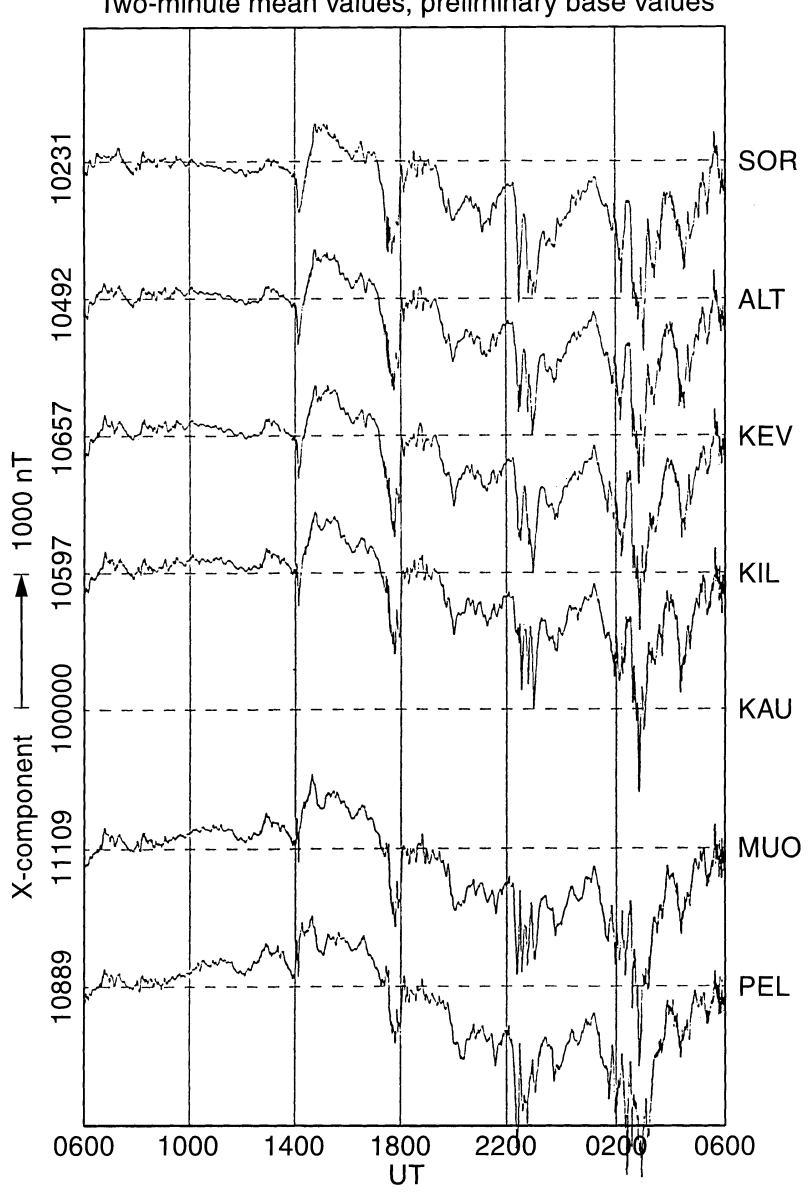

Fig. 8. EISCAT magnetometer data (geographic $X$-component) on 23-25 March 1991

\subsection{Storm on 27 November 1990}

This event is a very good example of an appearance of a strong westward electrojet in the evening sector at all EISCAT magnetometer cross stations. The maximum of the negative bay occurring about at $1725 \mathrm{UT}$ was reached at approximately $1300 \mathrm{nT}$, as is shown in Fig. 7 on the left-hand side. The equivalent current system at this time is represented in Fig. 11, showing a midlatitude current system overlapped by a westward electrojet in auroral latitudes. The asymmetry at 1700-1900 UT (Fig. 3) is very high and shows a fairly good correlation to the appearance of the westward electrojet. However, in this case it is also possible that the asymmetry is connected with the mid-latitude current system. The $H$-component of Dombas is depicted in Fig. 14. At nearly 1700 UT we observe a strong positive bay in Dombas which has a sudden beginning characteristic of an explosive eastward electrojet.

\subsection{Storm on 25 March 1991}

The asymmetry in the late recovery phase (Fig. 4) for this event was investigated. The highest asymmetry at this time appeared at $1300 \mathrm{UT}$, when the intensity of the eastward electrojet was low (Fig. 8). During the maximum of the eastward-electrojet intensity the asymmetry diminished. Furthermore, at $1700 \mathrm{UT}$ we observed a well-pronounced current system at midlatitudes in the evening sector. This happened when the asymmetry was at a minimum. Therefore, this event too does not let us draw the conclusion that the lowlatitude magnetic-field asymmetry is connected with the current observed in mid-latitudes. Of course, in the case of this eastward electrojet we cannot confirm that this is an explosive one.

\section{Preliminary theoretical model}

To explain the differences in the time evolution of the ring current and the eastward electrojet we have focused in detail on processes in the Kamide and Fukushima (1972) electric circuit. Let us introduce a model of the three-dimensional current system (shown in Fig. 15) in which $I_{R}(t)$ is the magnetospheric partial ring current in the afternoon/evening sector, and $I_{E}(t)$ is the auroral eastward electrojet. A symmetric current loop exists in the southern hemisphere.

Really, the position of the field-aligned currents depends on the kinetic processes of the ring-current ion interaction in the magnetosphere and the ionosphere. For instance, the cyclotron interaction of the ringcurrent particles with ion cyclotron turbulence near the evening plasmaspheric bulge boundary is responsible for the position of the incoming field-aligned currents into the ionosphere (Grafe et al., 1996). However, the main focus of our interest is the rather slow process in the electric circuit. The quasi-static assumption is sufficient for analysis of this type of process because the characteristic wave length of events is much greater than the magnetospheric large scale. Finally, taking this into consideration we get the simple electric circuit model as shown in Fig. 16.

Here, $C$ is the condenser value between the condenser plates 1 and 2 and $R$ is the active resistivity of the circuit. There is a slightly more general form of equivalent scheme with the circuit inductivity $L_{C}$ in the appendix. This general form results from model problem investigations. It is interesting to point out that this inductivity depends not only on the circuit inductivity but also on the gyrotropy of plasma inside the current loop. However, for a time-scale of processes studied in this work the term of the inductivity is highly limited, and we do not present this element in Fig. 16. The order of the magnitude of the condenser value will be determined by means of the well-known formula

$C=\frac{\varepsilon_{0} \varepsilon_{\perp} S}{d}$.

Here $\varepsilon_{0}=9 \cdot 10^{-12} \mathrm{~A} \mathrm{~s} / \mathrm{Vm}$ is the absolute dielectric constant, $\varepsilon_{\perp}$ is the transverse component of the dielectric tensor which is equal to $\varepsilon_{\perp}=1+c^{2} / V_{A}^{2} \approx c^{2} / V_{A}^{2}$ for low-frequency processes, where $c$ and $V_{A}$ are the light and the Alfvén velocities, $S$ is the size of the surface of 

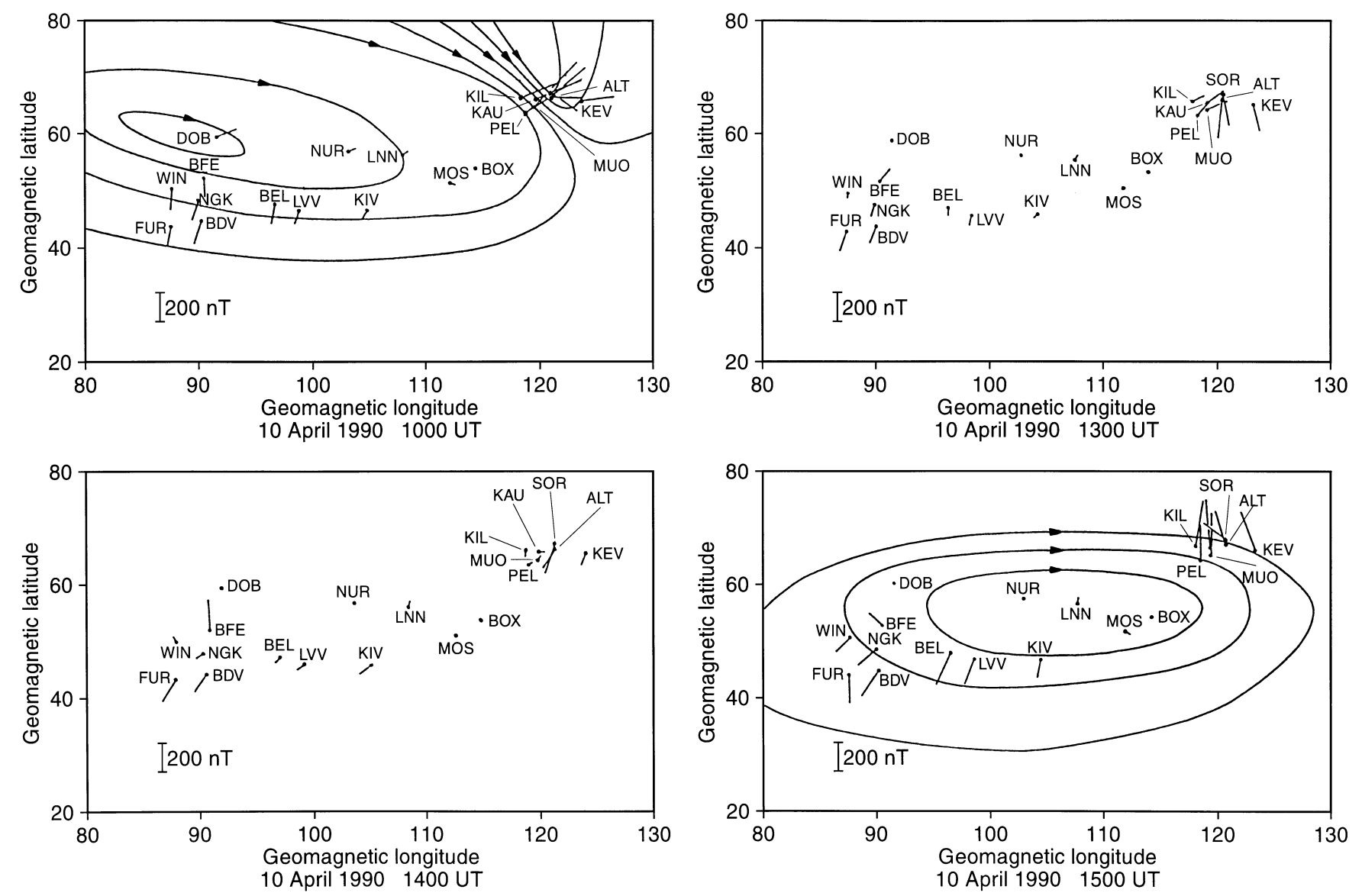

Fig. 9. Horizontal magnetic disturbance vectors and the equivalent ionospheric current system, respectively, for different time moments on 10 April 1990

the condenser plate 1 being close to $S \approx r_{0}^{2} L \Delta L$ for dipole magnetic field geometry, where $r_{0}$ is the earth radius, $d$ is the distance between the two condenser plates 1 and 2 and $d \approx r_{0} L \Delta \lambda / 360^{\circ}$, where $\Delta \lambda$ is the longitudinal distance between the surfaces 1 and 2 in degree (for typical magnetospheric conditions $\varepsilon_{\perp} \approx 10^{5}, L \approx 3$, $\left.\Delta L \approx 1, \Delta \lambda \approx 30^{\circ}\right)$. So we get

$C=\varepsilon_{0} \varepsilon_{\perp} r_{0} \Delta L\left(360^{\circ} / \Delta \lambda\right) \approx 60 \mathrm{~F}$.

The active circuit resistivity $R$ really depends on many factors and the value is significantly greater than what we can expect from the analysis of the ionospheric Pedersen and Hall conductivity. It is possible to define the real resistivity by the energy balance equation of the partial ring current,

$\frac{\mathrm{d} W}{\mathrm{~d} t}=R I_{E}^{2}$

where $W$ is the ring current energy. Taking into account that the characteristic partial ring current decay time $T_{R} \approx 4 \cdot 10^{3} \mathrm{~s}$, and $W \approx 2 \cdot 10^{15} \mathrm{~J}$ (Feldstein et al., 1986), and $I_{E} \approx 10^{5} \mathrm{~A}$, we can postulate the following approximation:

$R \approx \frac{W}{T_{R} I_{E}^{2}} \approx 50 \Omega$

Finally we get the characteristic time-scale of the circuit
$\tau=R C \approx 3 \cdot 10^{3} \mathrm{~s}$.

The evolution of the eastward electrojet in the framework of the equivalent scheme of Fig. 16 is determined by the equation

$\tau \frac{\mathrm{d} I_{E}}{\mathrm{~d} t}+I_{E}=I_{R}$.

The solution of this equation has the following form:

$I_{E}(t)=\frac{1}{\tau} \int_{0}^{t} I_{R}\left(t^{\prime}\right) \exp \left(\frac{t^{\prime}-t}{\tau}\right) \mathrm{d} t^{\prime}$.

In accordance with this solution only variations averaged over the time-scale $\tau$ of the partial ring current can exist in the eastward electrojet. The higher frequency variations of the partial ring current pass through the condensor.

\section{Discussion}

The observations show clearly that the low-latitude magnetic-field asymmetry is connected rather to the westward than to the eastward electrojet. In the end no relationship to the convection-like eastward electrojet could be found, in contradiction to the conception of Kamide and Fukushima (1972). They assume that just this eastward electrojet which is part of the twin vortex 

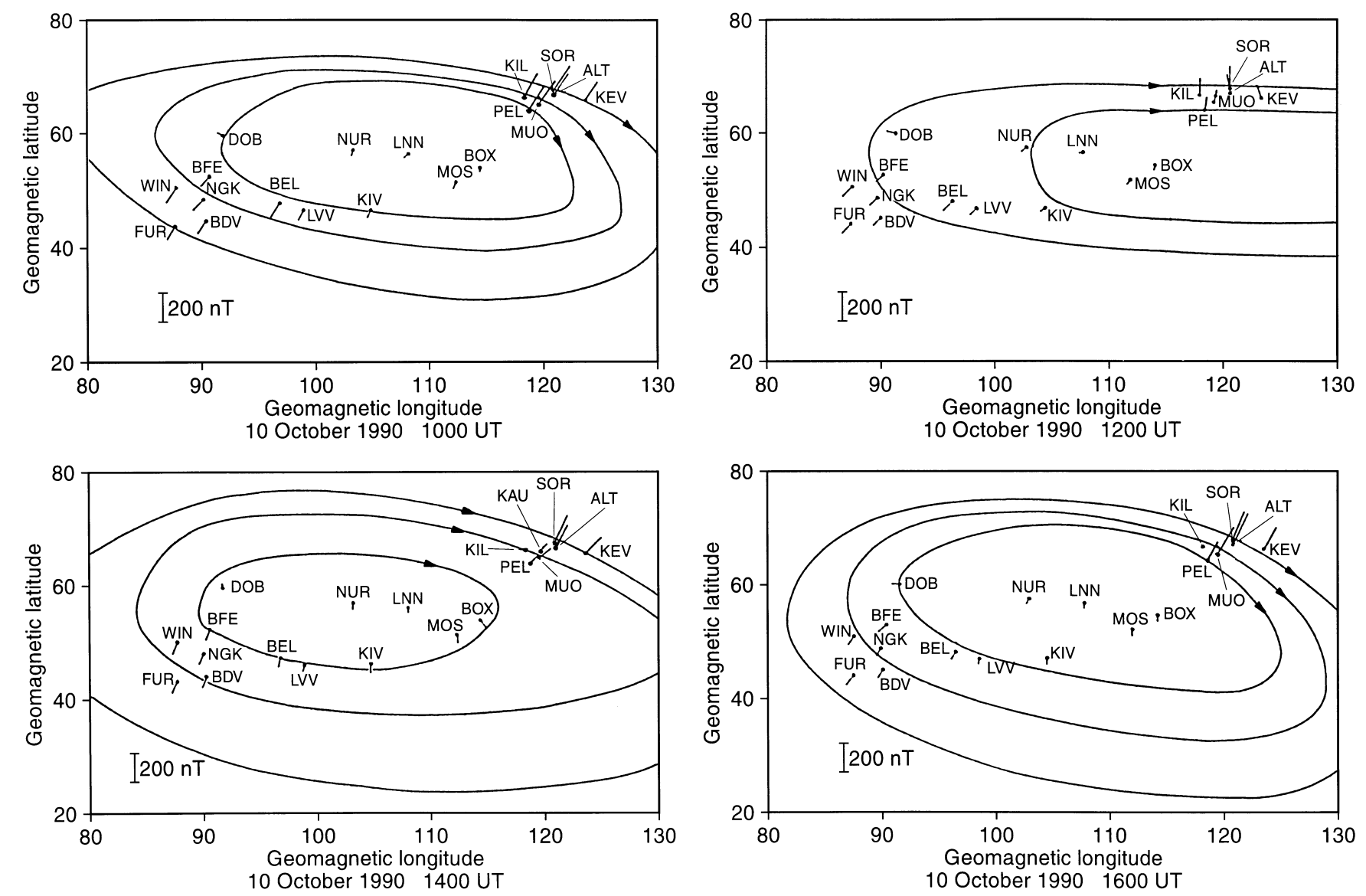

Fig. 10. Horizontal magnetic disturbance vectors and the equivalent ionospheric current system, respectively, for different time moments on 10 October 1990

current system caused by convection is connected to the partial ring current of the same three-dimensional current system. While it is very easy to imagine such a current system, the observations here do not correspond to the idea. Grafe (1977) has already shown that the correlation between ASY and AL is better than between ASY and AU. On the other hand it is rather difficult to

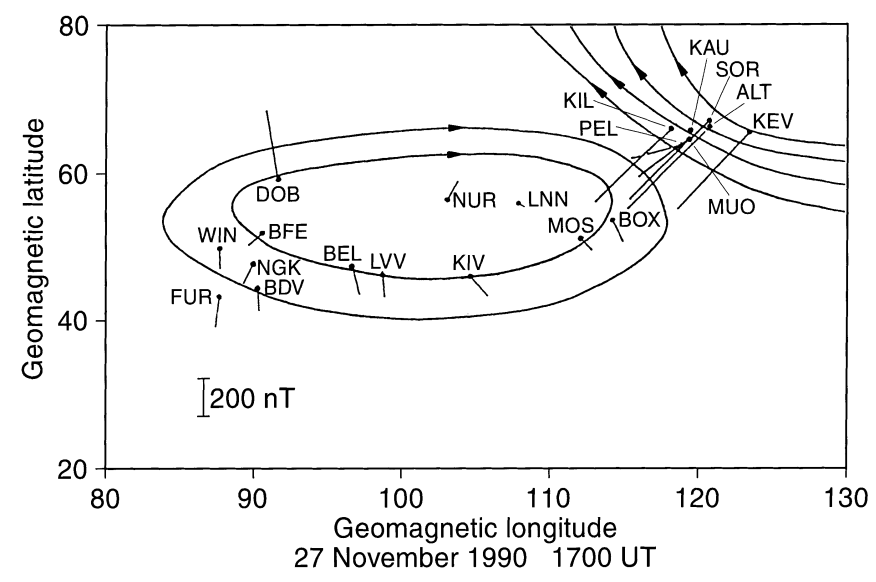

Fig. 11. Horizontal magnetic disturbance vectors and the equivalent ionospheric current system, respectively, for 1700 UT on 27 November 1990 imagine a common three-dimensional current system between a westward-flowing electrojet and a westwardflowing asymmetric ring current. In the case of the storm on 27 November 1990 we have seen that the strong asymmetry was closely correlated to an explosive eastward electrojet appearing equatorwards from a strong explosive westward electrojet. This gives us reason to suppose that perhaps an explosive eastward auroral electrojet is the characteristic feature of a partial ring current three-dimensional current system. However, Fig. 13 has clearly shown a close correlation between asymmetry and the westward electrojet intensity. On the other hand, we have to take into account that during active intervals the eastward electrojet moves towards the equator. Therefore, we cannot always see its activation in the AU indices. However, at the time of 1400 UT nor did any eastward-electrojet activity in subauroral and mid-latitudes exist, as shown in Fig. 9.

Consequently, the observations investigated are ambiguous in their interpretation. On one hand the close correlation between ASY and westward-electrojet intensity is difficult to describe by a three-dimensional current system, and on the other the missing correlation between ASY and eastward-electrojet intensity leads to doubts about a common three-dimensional current system as proposed by Kamide and Fukushima (1972). We at first had the idea that ASY is connected with the explosive 

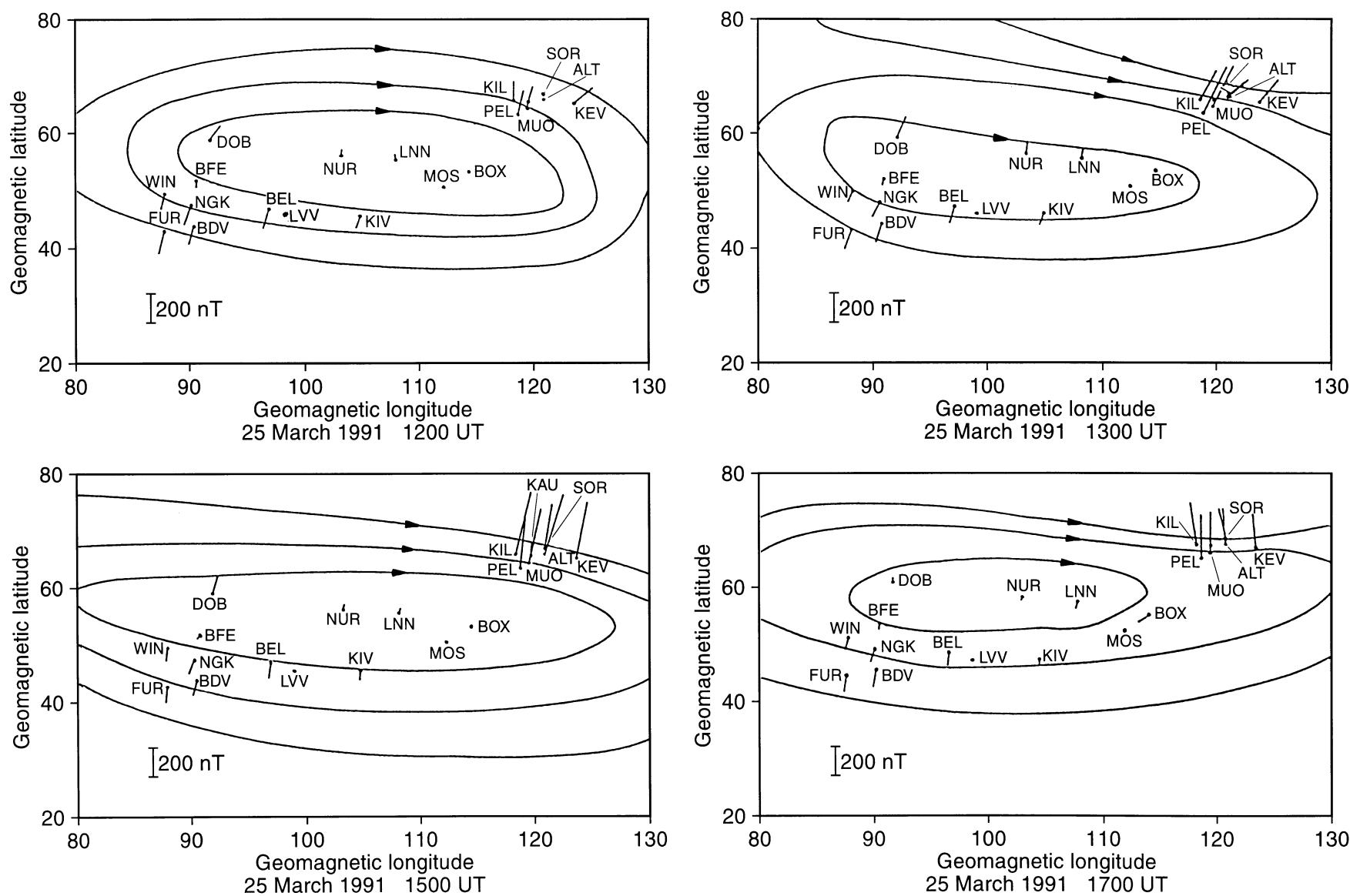

Fig. 12. Horizontal magnetic disturbance vectors and the equivalent ionospheric current system, respectively, for different time moments on 25 March 1991

eastward electrojet; however, now we must agree that the observations do not confirm this supposition.

We could find a model explaining observed differences in the time evolution in the magnetic field of the ring current and of the eastward electrojet. By accepting this model we would not be compelled to leave the idea of a

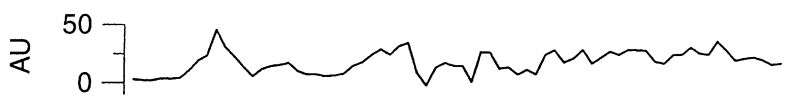

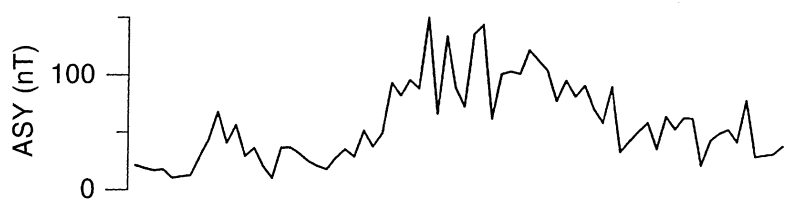

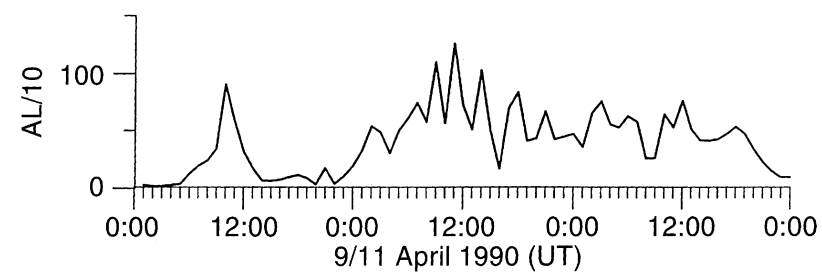

Fig. 13. Comparison between the electrojet indices (AL and AU) and ASY on 9-11 April 1990 common three-dimensional current system for the asymmetric ring current and the eastward electrojet. It is necessary to point out that the magnetic field of the ring current does not only depend on the current intensity, but also on its distribution in the space and position of the current. Moreover, we have no exact information of these values. It is possible that these values also have a characteristic time modulation. However, in our analysis we assume that these values are stable. Furthermore, we believe that the condenser

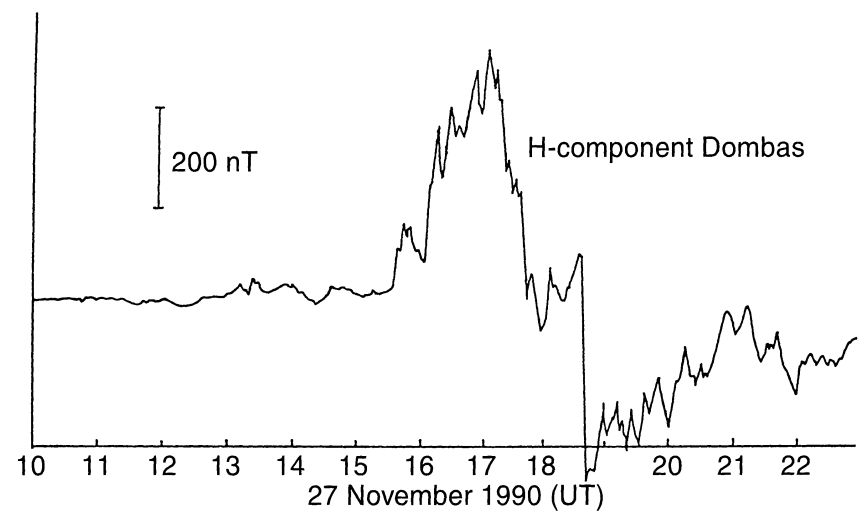

Fig. 14. $H$-component of Dombas at afternoon and evening on 27 November 1990 


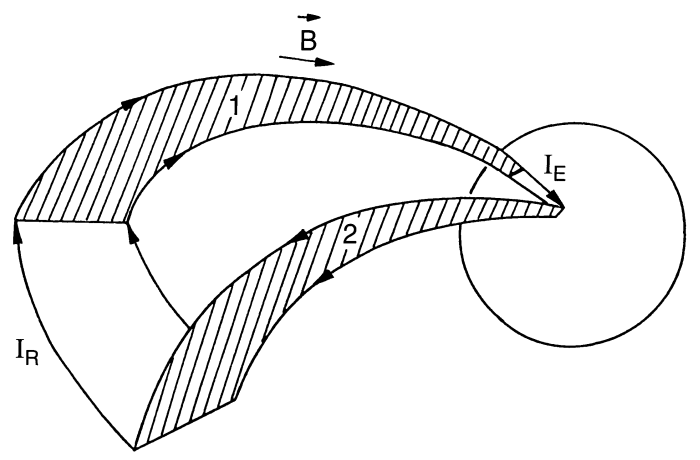

Fig. 15. Sketch of a three-dimensional ionospheric-magnetospheric current system

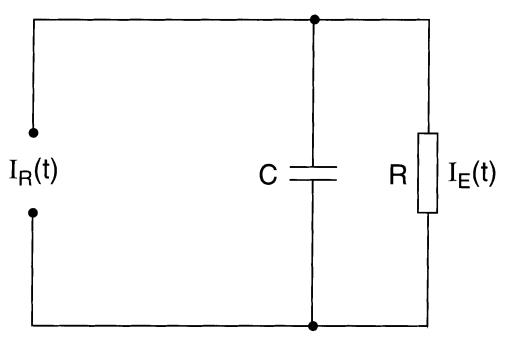

Fig. 16. Idealized circuit of the current system shown in Fig. 11

effect plays an important role in the global electric circuit. Using this simple model it is possible to explain why the eastward electrojet changes more smoothly in time in comparison to the ring current.

We do not exactly know the sources of the ring current, but it is clear that its time variation also depends on peculiarities of the tail convection process (Williams, 1981). This convection is ordinarily discussed as a major source of the convection westward electrojet (Kamide, 1982). The corresponding current loop has another characteristic time-scale. This can help to understand why the westward electrojet has a similar time variation as the asymmetric ring current. However, this is an extensive unsolved problem and important for further investigations.

Furthermore, there are some additional problems for further analysis. It is necessary to compare the current evolution with observations of the electric fields in the ionosphere and the magnetosphere. We have additional reasons for studying various resistivity processes in the current loop. This becomes necessary because the real resistivity value $R$ is more than 100 times greater than the value obtained from the ionospheric Pedersen and Hall conductivity. It is also interesting to note that the eigenfrequency of the loop $\Omega=1 \sqrt{L C}$ corresponds to a period of about $60 \mathrm{~s}$.

Acknowledgements. The authors are indebted to the IMAGE team for the magnetometer chain data and all magnetic observatories which have sent us copies of magnetograms. One of the authors (P. A. Bespalov) wishes to acknowledge the GeoForschungsZentrum Potsdam for the support of the work visit in the Niemegk Observatory.

Topical Editor K.-H. Glaßmeier thanks two referees for their help in evaluating this paper.

\section{Appendix. Three-dimensional condenser model}

Let us introduce the three-dimensional current system in Fig. 17. In this model $I_{R}(t)$ is the magnetospheric partial ring current, $I_{E}(t)$ is the eastward-electrojet current, $I_{x}$ and $I_{y}$ are currents across magnetic field $\mathbf{B}, I_{N}$ is the northward ionospheric current, $l_{x}, l_{y}$ and $l_{z}$ are sizes of the flux tube with the eastward electrojet in the food, and $h$ is the electrojet thickness. Hereby we take into account the very high conductivity along the magnetic field, and that these lines are equipotentials. Since the purpose of our analysis is only a preliminary investigation of the problem in this simplified model, we do not bear in mind the dipole geometry of the magnetic field. Practically, results of the investigation show the necessity to correct formulas for more real geometry of the magnetic field.

Our aim is to investigate the rather slow processes in the electric circuit. For the analysis of this kind of process, the quasi-static assumption was used because the characteristic wave length of events is much larger than the magnetospheric size scale. The complete set of quasi-static equations for the magnetospheric part of the circuit is based on Poisson's equation in the anisotropic plasma and the charge conserve equation

$$
\begin{gathered}
\operatorname{div}(\varepsilon \operatorname{grad} \varphi)=-\frac{\rho}{\varepsilon_{0}}, \\
\operatorname{div} \mathbf{j}=-\frac{\partial \rho}{\partial t},
\end{gathered}
$$

where $\varphi$ is the electric potential, $\rho$ is the change density, $\boldsymbol{j}$ is the electric current density, $\varepsilon_{0}$ is the absolute dielectric constant and the plasma dispersion characteristic is determined by the well-known dielectric tensor (FrankKamenezki, 1967)

$\varepsilon=\left[\begin{array}{ccc}\varepsilon_{\perp} & i g & 0 \\ -i g & \varepsilon_{\perp} & 0 \\ 0 & 0 & \varepsilon_{l}\end{array}\right]$

which has the above form in the frame with $z$-axis parallel to the magnetic field. Equation A1 for $E_{z}=0$ ( $E_{z}$ is the electric field along the magnetic field) has the following form

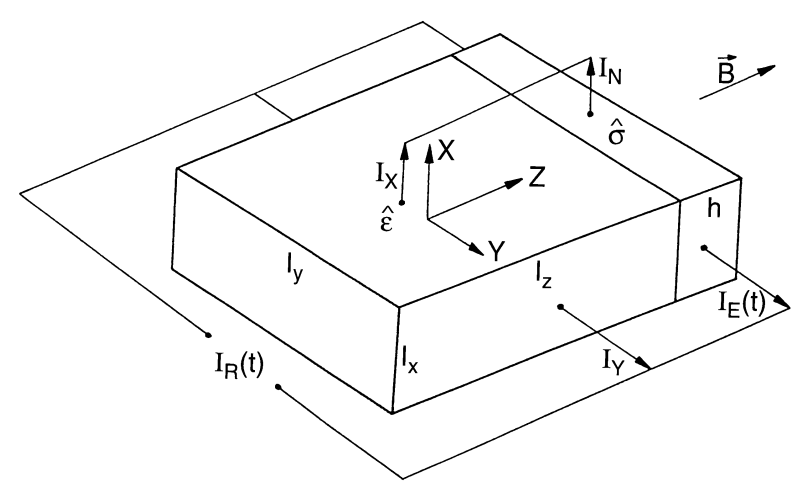

Fig. 17. Sketch of the condenser model of the current system shown in Fig. 11 
$\frac{\partial}{\partial x}\left(\varepsilon_{\perp} \frac{\partial \varphi}{\partial x}+i g \frac{\partial \varphi}{\partial y}\right)+\frac{\partial}{\partial y}\left(-i g \frac{\partial \varphi}{\partial x}+\varepsilon_{\perp} \frac{\partial \varphi}{\partial y}\right)=-\frac{\rho}{\varepsilon_{0}}$,

$I=\frac{\mathrm{d} Q_{y}}{\mathrm{~d} t}$,

where $Q_{y}$ is the electric charge value on the boundary surface, which is normal to the $y$-axis. Let us reduce now Eq. (A3a) to integral form. For this purpose this equation acts on

$\frac{l_{z}}{2} \frac{l_{x}}{2} \frac{l_{y}}{2} y^{\prime}$

by means of the operation $\int \mathrm{d} z \int \mathrm{d} x \int \mathrm{d} y \int \mathrm{d} y^{\prime} \cdots$

$-\frac{l_{z}}{2}-\frac{l_{x}}{2}-\frac{l_{y}}{2}-\infty$

where $\pm l_{x} / 2, \pm l_{y} / 2, \pm l_{z} / 2$ are parallelepiped boundary positions. As a result, we get instead of Eq. (A3):

$-i g \varepsilon_{0} l_{z} U_{x}+\frac{\varepsilon_{0} \varepsilon_{\perp} l_{x} l_{z}}{l_{y}} U_{y}=Q_{y}$

$I_{y}=\frac{\mathrm{d} Q_{y}}{\mathrm{~d} t}$,

where $U_{x}$ and $U_{y}$ are the electric voltages between the appropriate surfaces. We do not introduce into the system Eq. (A4) an equation in the $x$-direction similar to part a because the parameter $|g| \ll 1$, and we can take into account the gyrotropy factor only as a small additional factor in the complete analysis. Let us discuss the ionospheric part of the current circuit. As is well known, the low-frequency part of the ionospheric current density is equal to

$\mathbf{j}=\sigma_{P} \mathbf{E}+\frac{\sigma_{H}}{B} \mathbf{E} \times \mathbf{B}$,

where $\sigma_{P}$ and $\sigma_{H}$ are Pedersen and Hall conductivities and $\mathbf{E}$ is the ionospheric electric field. We have from Eq. (A5) the expression for the total ionospheric electric currents

$$
\begin{aligned}
& I_{E}=\left(\sigma_{P} E_{y}-\sigma_{H} E_{x}\right) h l_{x}, \\
& I_{N}=\left(\sigma_{P} E_{x}+\sigma_{H} E_{y}\right) h l_{y},
\end{aligned}
$$

where $h$ is the ionospheric thickness. The eastwardelectrojet electric current is substantially larger than the northward-electrojet electric current. Taking this into account we can assume in Eq. (A6) that $I_{N}=0$ and:

$I_{E}=\left(\sigma_{P}+\frac{\sigma_{H}^{2}}{\sigma_{P}}\right) \frac{h l_{x}}{l_{y}} U_{y}, \sigma_{P} U_{x} l_{y}=\sigma_{H} U_{y} l_{x}$,

In this way we have the complete set of equations from Eqs. (A4) and (A7):

$-i g \varepsilon_{0} l_{z} U_{x}+C U_{y}=Q_{y}$,

$I_{y}=\frac{\mathrm{d} Q_{y}}{\mathrm{~d} t}$,

$I_{E}=\frac{U_{y}}{R}$,

$$
\begin{aligned}
& \sigma_{P} U_{x} l_{y}=\sigma_{H} U_{y} l_{x}, \\
& I_{R}=I_{E}+I_{y},
\end{aligned}
$$

where the last equation follows from Fig. 13 and

$C=\frac{\varepsilon_{0} \varepsilon_{\perp} l_{x} l_{z}}{l_{y}}, \quad R=\frac{l_{y}}{h l_{x}}\left(\sigma_{P}+\frac{\sigma_{H}^{2}}{\sigma_{P}}\right)^{-1}$.

For low-frequency processes in the plasma (FrankKamenezki, 1967) we get

$\varepsilon_{\perp}=1+\frac{c^{2}}{v_{A}^{2}}, \quad g=\frac{\omega m_{i} c^{2}}{\omega_{B i} m_{e} v_{A}^{2}}$

where $c$ is the speed of light, $v_{A}$ is the Alfvén velocity and $m_{e}$ and $m_{i}$ are the electron and ion masses, respectively. Equation (A9) is valid for $\exp (-i \omega t)$ processes. From Eq. (A8) results

$$
\begin{gathered}
L_{g} \frac{\mathrm{d} U_{y}}{\mathrm{~d} t}+C U_{y}=Q_{y}, \\
I_{y}=\frac{\mathrm{d} Q_{y}}{\mathrm{~d} t}, \\
I_{E}=\frac{U_{y}}{R}, \\
I_{R}=I_{E}+I_{y},
\end{gathered}
$$

where

$L_{g}=\frac{m_{i} c^{2} \varepsilon_{0} \sigma_{H} l_{x} l_{z}}{m_{e} \omega_{B i} v_{A}^{2} \sigma_{P} l_{y}}$.

Finally, it is easy to obtain Eq. (A11) from the system of equations of Eq. (A10)

$R L_{C} \frac{\mathrm{d}^{2} I_{E}}{\mathrm{~d} t^{2}}+R C \frac{\mathrm{d} I_{E}}{\mathrm{~d} t}+I_{R}=I_{R}$

Here $L_{C}=L_{g}+L_{*}$, where $L_{g}$ is the above mentioned indication determined by gyrotropy, $L_{*}$ is the simple circuit induction. There are two eigentimes for the current circuit

$\Omega=L_{C} C^{-1 / 2}, \quad \tau=R C$,

where $\Omega$ is the circuit eigenfrequency and $\tau$ is the characteristic circuit time-scale.

\section{References}

Baumjohann, W., Some recent progress in substorm studies, J. Geomagn. Geoelectr., 38, 633, 1986.

Clauer, C. R., R. L. McPherron, C. Searls, and M. G. Kivelson, Solar wind control of auroral zone geomagnetic activity, Geophys. Res. Lett., 8, 915, 1981.

Crooker, N. U., and G. L. Siscoe, Birkeland currents as the cause of the low-latitude asymmetric disturbance field, J. Geophys. Res., 86, 11201, 1981.

Feldstein, Y. I., A. E. Levitin, V. Y. Pisarsky, N. M. Rudneva, A. Grafe, P. Ochabova, and A. Prigancova, Energy regimes in the earth's magnetosphere, Stud. Geophys. Geod., 30, 268, 1986.

Frank-Kamenezki, D. A., Vorlesungen über Plasmaphysik, 119, VEB Deutscher Verlag der Wissenschaften, 1967.

Grafe, A., On the coupling of the asymmetric ring current and the eastward electrojet, Gerl. Beitr. Geophys., 86, 265, 1977. 
Grafe, A., The explosive character of the eastward electrojet during the substorm event on April 1, 1986, Gerl. Beitr. Geophys., 99, 539,1990

Grafe, A., Freja electron precipitation as a hint for an explosive development of the eastward electrojet. In Proc. Sec. Int. Conf. Substorms, Fairbanks, Alaska, Ed. p.391, 1994.

Grafe, A., V. Yu. Trakhtengerts, P. A. Bespalov, and A. G. Demekhov, The evolution of the low-latitude geomagnetic storm field and the importance of turbulent diffusion for ring current particles losses, J. Geophys. Res., 101, 24689, 1996.

Harel, M., R. A. Wolf, R. W. Spiro, P. H. Reiff, C. K. Chen, W. J. Burke, F. J. Rich, and M. Smiddy, Quantitative simulation of a magnetospheric substorm, 2, comparison with observations, J. Geophys. Res., 86, 2242, 1981.

Kamide, Y., The two-component auroral electrojet, Geophys. Res. Lett., 9, 1175, 1982.

Kamide, Y., and N. Fukushima, Positive geomagnetic bay in evening high latitudes and their possible connection with partial ring current, Rep. Ionos. Space Res. Jap., 26, 79, 1972.
Kamide, Y., and J. E. Vickrey, Relative contribution of ionospheric conductivity and electric field to the auroral electrojets, J. Geophys. Res., 88, 7989, 1983.

Kawasaki, K., and S.-I. Akasofu, Low-latitude DS component of geomagnetic storm field, J. Geophys. Res., 76, 2396, 1971.

Lühr, H., S. Thürey, and N. Klöcker, The EISCAT magnetometer cross: operational aspects, first results, Geophys. Surv., 6, 305, 1984.

Pytte, T., R. L. McPherron, E. W. Hones, and H. I. West, Multiple satellite studies of magnetic substorms. Distinction between polar magnetic substorms and convection-driven negative bays, J. Geophys. Res., 83, 663, 1978.

Sergeev, V. A., On the classification of magnetospheric phenomena, Phys. Solariterr., 4, 19, 1977.

Williams, D. J., Ring current composition and sources: an update, Planet. Space Sci., 11, 1195, 1981. 